\begin{tabular}{lll}
\hline VOL. XXXIX & 1978 & FASC. 2
\end{tabular}

\title{
ON A LOWER BOUND OF THE SECOND EIGENVALUE \\ OF THE LAPLACIAN ON AN EINSTEIN SPACE
}

BY

\section{SHUKICHI TANNO (SENDAI)}

0. Introduction. Let $(M, g)$ be a Riemannian manifold and let $\Delta$ be the Laplacian acting on $C^{\infty}$-functions on $(M, g)$. Then some results on lower bounds or upper bounds of the first eigenvalue of $\Delta$ are given in several situations (cf., e.g., [1]-[4] and [7]).

Let $(M, g)$ be a compact orientable Einstein space with constant scalar curvature $S=m(m-1) K$, where $m=\operatorname{dim} M \geqslant 2$. Then the lower bound of the first eigenvalue $\lambda_{1}$ of $\Delta$ is $m K$. Furthermore, if $(M, g)$ admits an eigenfunction $f(\Delta f=-m K f)$ corresponding to $m K$, then $(M, g)$ is isometric to a Euclidean $m$-sphere of constant curvature $K$ (cf. Obata [6]).

In this paper* we prove the following theorem:

THEOREM. Let $(M, g)$ be a compact orientable Einstein space with constant positive scalar curvature $S=m(m-1) K$, where $m=\operatorname{dim} M \geqslant 2$. Let $B$ be the minimum of the sectional ourvature of $(M, g)$ and assume that B. $>$ 0. Then

(i) there is no eigenvalus $\lambda$ of $\Delta$ such that

$$
m K<\lambda \leqslant K+2(m-1) B
$$

(ii) if $B \neq K$, then the first eigenvalue $\lambda_{1}$ of $\Delta$ satisfies

$$
\lambda_{1}>K+2(m-1) B
$$

(iii) generally, the second eigenvalue $\lambda_{2}$ of $\Delta$ satisfies

$$
\lambda_{2}>K+2(m-1) B \text {. }
$$

It may be remarked that if $(M, g)$ is a Euclidean $m$-sphere of constant curvature $K$, then the second eigenvalue $\lambda_{2}$ of $\Delta$ is $2(m+1) K=K+$ $+(2 m+1) K$.

* This paper was done while the author was a guest at Teohnische Universität Berlin by DAAD.JSPS exchange program. 
The author is grateful to Professors D. Ferus and U. Simon. This paper is originated by Simon's paper [8].

1. Proof of the Theorem. Let $(M, g)$ be a compact orientable Einstein space stated in the Theorem. Let $f$ be a non-constant eigenfunction of $\Delta$ such that

$$
\Delta f=-\lambda f,
$$

where $\Delta f=g^{i j} \nabla_{i} \nabla_{f} f=\nabla^{i} \nabla_{i} f$, and $\nabla$ denotes the Riemannian connection defined by $g=\left(g_{i j}\right)$. By Obata's theorem in the introduction, we study only the case where $\lambda>m K$. We put

$$
f_{i}=\nabla_{i} f \quad \text { and } \quad f_{i j}=\nabla_{j} \nabla_{i} f \text {. }
$$

Now we define a $(0,2)$-tensor field $A=\left(A_{i j}\right)$ by

$$
A_{i j}=f_{i j}+K f g_{i j} \text {. }
$$

Then we get $\nabla_{k} A_{i j}=\nabla_{k} f_{i j}+K f_{k} g_{i j}$ and

where

$$
\begin{aligned}
\nabla_{k} A_{i j} \nabla^{k} A^{i j} & =\nabla_{k} f_{i j} \nabla^{k} f^{i j}+2 K f^{k} \nabla_{k} \Delta f+K^{2} m f_{k} f^{k} \\
& =\nabla_{k} f_{i j} \nabla^{k} f^{i j}+\left(m K^{2}-2 K \lambda\right) f_{k} f^{k},
\end{aligned}
$$

$$
\nabla_{k} f_{i j} \nabla^{k} f^{i j}=\nabla_{k}\left(f_{i j} \nabla^{k} f^{i j}\right)-f_{i j} \nabla_{k} \cdot \nabla^{k} f^{i j}
$$

By applying the Ricci identity and using the Einstein condition, the second term of the right-hand side is calculated to be

$$
-f_{i j} \nabla_{k} \nabla^{k} f^{i j}=-f^{i j} \nabla_{j} \nabla_{i} \Delta f+2 f^{i j}\left(R_{k j}^{h}{ }^{k} f_{i h}+R^{h}{ }_{i j}^{k} f_{h k}\right),
$$

where $\left(R_{j k l}^{i}\right)$ denotes the Riemannian curvature tensor field (cf. [8]).

At each point $x$ of $M$, we take an orthonormal frame $\left\{E_{i}\right\}$ such that each $E_{i}$ is an eigenvector of $\left(f_{j}^{i}\right)$ at $x$ and let $\sigma_{i}$ be the corresponding eigenvalue. We estimate scalars with respect to this frame $\left\{\boldsymbol{E}_{i}\right\}$. Then

$$
\begin{aligned}
& -f^{i j} \nabla_{j} \nabla_{i} \Delta f=\lambda f^{i j} f_{i j}=\lambda \sum_{i}\left(\sigma_{i}\right)^{2} \\
& =\frac{\lambda}{m}\left\{\sum_{i<j}\left(\sigma_{i}-\sigma_{j}\right)^{2}+\left(\sum_{i} \sigma_{i}\right)^{2}\right\}=\frac{\lambda}{m}\left\{\sum_{i<j}\left(\sigma_{i}-\sigma_{j}\right)^{2}+(\lambda f)^{2}\right\} .
\end{aligned}
$$

On the other hand, we get (cf. [8])

$$
2 f^{i j}\left(R_{k j}^{h}{ }^{k} f_{i h}+R^{h}{ }_{i j}^{k} f_{h k}\right)=-2 \sum_{i<j} K_{i j}\left(\sigma_{i}-\sigma_{j}\right)^{2}
$$

where $K_{i j}$ denotes the sectional curvature for a 2-plane spanned by $E_{i}$ and $\boldsymbol{E}_{j}$. 
Next we rewrite $f^{i j} f_{i j}$ and $f_{k} f^{k}$ :

$$
\begin{aligned}
f^{i j} f_{i j} & =\nabla_{j}\left(f_{i} f^{i j}\right)-f^{i} \nabla_{j} \nabla_{i} f_{j} \\
& =\nabla_{j}\left(f_{i} f^{i j}\right)-f^{i}\left(V_{i} \Delta f+(m-1) K f_{i}\right) \\
& =\nabla_{j}\left(f_{i} f^{i j}\right)+(\lambda-m K+K) f_{k} f^{k}, \\
f_{k} f^{k} & =\nabla_{k}\left(f f^{k}\right)-f \Delta f=\nabla_{k}\left(f f^{k}\right)+\lambda f^{2} .
\end{aligned}
$$

By (7) and (8) we get

$$
\begin{aligned}
f^{i j} f_{i j} & =\nabla_{j}\left(f_{i} f^{i j}+(\lambda-m K+K) f f^{j}\right)+(\lambda-m K+K) \lambda f^{2} \\
& =\nabla_{j} N_{1}^{j}+(\lambda-m K+K) \lambda f^{2},
\end{aligned}
$$

where $N_{1}$ (or $N_{2}$ and ' $N_{3}$, later) denotes some vector field on $M$ the explicit expression of which we do not need. Eliminating $f^{i j} f_{i j}$ from (5) and (9), we obtain

$$
\lambda f^{2}=\nabla_{j} N_{2}^{j}+\frac{1}{m(\lambda-m K+K)-\lambda} \sum_{i<j}\left(\sigma_{i}-\sigma_{j}\right)^{2} .
$$

Applying (4)-(10), we can write (3) as

$$
\nabla_{k} A_{i j} \nabla^{k} A^{i j}=\nabla_{j} N_{3}^{j}+\sum_{i<j}\left\{\frac{\lambda-K}{m-1}-2 K_{i j}\right\}\left(\sigma_{i}-\sigma_{j}\right)^{2} .
$$

Therefore, if $\lambda \leqslant K+2(m-1) B$, then integrating (11) on $M$ we obtain $\nabla_{k} A_{i j}=0$. Since $(M, g)$ is irreducible by $B>0, A_{i j}$ is of the form $A_{i j}=c g_{i j}$ for some constant $c$, and

$$
f_{i j}+K f g_{i j}=c g_{i j} \text {. }
$$

Transvecting the last equation with $g^{i j}$; we get

$$
\Delta f+m \boldsymbol{K} f=m c \text {. }
$$

Since $f$ is an eigenfunction, integrating the last equation on $M$ we get $c=0$ and $\lambda=-m K$. This is a contradiction. Therefore, there is no eigenvalue $\lambda$ such that $m K<\lambda \leqslant K+2(m-1) B$. This proves (i) of the Theorem. (ii) follows from (i) and Obata's theorem. And, generally, we have (iii).

\section{REFERENOES}

[1] I. Chavel and E. A. Feld man, The first eigenvalue of the laplacian on manifolds of non-negative curvature, Compositio Mathematioa 29 (1974), p. 43-53.

[2] J. Cheөger, The relation between the Laplacian and the diameter for manifolds of non-negative curvature, Archiv der Mathematik 19 (1968), p. 558-560.

[3] - A lower bound for the smallest eigenvalue of the Laplacian, Problem in Analysis (Bochner), Princeton 1970, p. 175-199. 
[4] E. Mazet, Une majoration de $\lambda_{1}$ du type de Cheeger, Comptes Réndus Hebdomadaires des Séances de l'Académie des Sciences (Paris) 277 (1973), p. 171-174.

[5] T. Nagano, On the minimum eigenvalue of the Laplacians in Riemannian manifolds, Scientifio Papers of the College of General Eduoation, University of Tokyo, 11 (1961), p. 177-181.

[6] M. Obata, Certain conditions for a Riemannian manifold to be isometric with a sphere, Journal of the Mathematical Society of Japan 14 (1962), p. 333-340.

[7] J.-C. Paris, Caloul d'un majorant de $\lambda_{1}$ en fonction d'un minorant de la courbure de Ricci, d'après la méthode de Oheeger, Comptes Rendus Hebdomadaires des Séances de l'Académie des Sciences (Paris) 279 (1974), p. 515-617.

[8] U. Simon, Isometries with spheres, Mathematische Zeitsohrift 153 (1977), p. 23-27.

TEOHNISOHE UNIVERSITAT BERLIN

FACHBEREICH 3, MATHEMATIK, BERLIN

MATHEMATICAL INSTITUTH

TOHOKU UNIVERSITY, SENDAI

Reçu par la Rédaction le 30.6.1976 